\title{
GLOBAL EXISTENCE OF STRONG SOLUTION TO 3D PERIODIC NAVIER-STOKES EQUATIONS
}

\author{
ABDELKERIM CHAABANI
}

\begin{abstract}
The purpose of this paper is to bring to light a method through which the global in time existence for arbitrary large in $H^{1}$ initial data of a strong solution to 3D periodic Navier-Stokes equations follows. The method consists of subdividing the time interval of existence into smaller sub-intervals carefully chosen. These sub-intervals are chosen based on the hypothesis that for any wavenumber $m$, one can find an interval of time on which the energy quantized in low-frequency components (up to $m$ ) of the solution $u$ is lesser than the energy quantized in high-frequency components (down to $m$ ) or otherwise the opposite. We associate then a suitable number $m$ to each one of the intervals and we prove that the norm $\|u(t)\|_{H^{1}}$ is bounded in both mentioned cases. The process can be continued until reaching the maximal time of existence $T_{\max }$ which yields the global in time existence of strong solution.
\end{abstract}

\section{INTRODUCTION}

Let us consider the following incompressible Navier-Stokes equations:

$$
\left\{\begin{array}{l}
\partial_{t} u-\nu \Delta u+(u \cdot \nabla) u+\nabla p=0, \quad(x, t) \in \mathbb{T}^{3} \times \mathbb{R}_{+} \\
\nabla \cdot u=0, \quad(x, t) \in \mathbb{T}^{3} \times \mathbb{R}_{+} \\
\left.u\right|_{t=0}=u_{0}(x), \quad x \in \mathbb{T}^{3}
\end{array}\right.
$$

where the constant $\nu>0$ is the viscosity of the fluid, and $\mathbb{T}^{3}=\mathbb{R}^{3} / \mathbb{Z}^{3}$ is the threedimensional torus with periodic boundary conditions. Here $u$ is a three-dimensional vector field $u=\left(u_{1}, u_{2}, u_{3}\right)$ representing the velocity of the fluid, and $p$ is a scalar denoting the pressure, both are unknown functions of the time variable $t$ and space variable $x$. We recall that the pressure can be eliminated by projecting (NSE) onto the space of free divergence vector fields, using the Leray projector

$$
\mathbb{P}=I d-\nabla \Delta^{-1} \nabla \cdot
$$

Thus, it will be convenient using the following equivalent system

$$
\left\{\begin{array}{l}
\partial_{t} u-\nu \Delta u+\mathbb{P}(u \cdot \nabla) u=0, \quad(x, t) \in \mathbb{T}^{3} \times \mathbb{R}_{+} \\
\nabla \cdot u=0, \quad(x, t) \in \mathbb{T}^{3} \times \mathbb{R}_{+} \\
\left.u\right|_{t=0}=u_{0}(x), \quad x \in \mathbb{T}^{3} .
\end{array}\right.
$$

We define the Sobolev spaces $H^{s}\left(\mathbb{T}^{3}\right)$ for $s \geq 0$ by the Fourier expansion

$H^{s}\left(\mathbb{T}^{3}\right)=\left\{u \in L^{2}\left(\mathbb{T}^{3}\right): u(x):=\sum_{k \in \mathbb{Z}^{3}} \hat{u}(k, t) e^{i k x}, \hat{u}(k, t)=\overline{\hat{u}(k, t)},\|u\|_{H^{s}}<\infty\right\}$,

2000 Mathematics Subject Classification. primary 35A01 secondary 35D35.

Key words and phrases. Navier-Stokes, global existence, strong solution. 
where

$$
\|u\|_{H^{s}}^{2}:=\sum_{k \in \mathbb{Z}^{3}}\left(1+|k|^{2 s}\right)|\hat{u}(k, t)|^{2}
$$

and

$$
\hat{u}(k, t)=\int_{\mathbb{T}^{3}} u(x) e^{-i k x} d x .
$$

We will also use the following function spaces:

$$
\begin{gathered}
\mathcal{D}_{\sigma}:=\left\{\varphi \in\left[C_{c}^{\infty}\left(\mathbb{T}^{3}\right)\right]^{3}: \nabla \cdot \varphi=0\right\} \\
L_{\sigma}^{2}\left(\mathbb{T}^{3}\right):=\text { closure of } \mathcal{D}_{\sigma} \text { in } L^{2} \\
H_{\sigma}^{1}\left(\mathbb{T}^{3}\right):=\text { closure of } \mathcal{D}_{\sigma} \text { in } H^{1}
\end{gathered}
$$

For an initial data $u_{0} \in L_{\sigma}^{2}\left(\mathbb{T}^{3}\right)$, it was proven by Leray and Hopf that there exists a global weak solution $u \in L_{t}^{\infty}\left(L_{\sigma}^{2}\right) \cap L_{t}^{2}\left(H_{\sigma}^{1}\right)$.

Theorem 1.1. For every $u_{0} \in L_{\sigma}^{2}\left(\mathbb{T}^{3}\right)$ there exists at least one global in time weak solution $u \in L^{\infty}\left(0, \infty ; L_{\sigma}^{2}\left(\mathbb{T}^{3}\right)\right) \cap L^{2}\left(0, \infty ; H_{\sigma}^{1}\left(\mathbb{T}^{3}\right)\right)$ of the Navier-Stokes equations satisfying the initial condition $u_{0}$. In particular, $u$ satisfies the energy inequality

$$
\frac{1}{2}\|u(t)\|_{L_{\sigma}^{2}\left(\mathbb{T}^{3}\right)}^{2}+\nu \int_{0}^{t}\|\nabla u(\tau)\|_{L_{\sigma}^{2}\left(\mathbb{T}^{3}\right)}^{2} d \tau \leq \frac{1}{2}\left\|u_{0}\right\|_{L_{\sigma}^{2}\left(\mathbb{T}^{3}\right)}^{2}
$$

This result was proved by Hopf [6] as a generalisation of a previous existence theorem due to Leray [8] for the whole space $\mathbb{R}^{3}$.

It is also known that local in time strong solutions exist on the whole space due to Leray [8], while the case of a bounded domain is due to Kiselev and Ladyzhenskaya $[7]$.

Theorem 1.2. There is a constant $C>0$ such that any initial condition $u_{0} \in$ $H_{\sigma}^{1}\left(\mathbb{T}^{3}\right)$ gives rise to a strong solution of the Navier-Stokes equations

$$
u \in L^{\infty}\left(0, T_{\max } ; H_{\sigma}^{1}\right) \cap L^{2}\left(0, T_{\max } ; H^{2}\right), \text { where } T_{\max }=\frac{C}{\left\|\nabla u_{0}\right\|_{L^{2}}^{4}} .
$$

The existence of global in time strong solution is known to occur for small initial data due to Fujita and Kato [5] and Chemin [2]. However, it remains the major open problem as to whether these solutions can be extended to be global in time for arbitrary large in $H^{1}$ initial data. Originally, the problem is the question of global existence of smooth solutions to the Navier-Stokes equations satisfying bounded energy condition (i.e.: $u \in C^{\infty}\left(\mathbb{R}_{+} \times \mathbb{T}^{3}\right)$ and $\left.\int_{\mathbb{T}^{3}}|u(x)|^{2} d x<\infty\right)$ or otherwise a breakdown. The official description has been given by Fefferman in [4]. The official Clay Millennium problem is to give a proof of one of the four following statements:

(A) Existence and smoothness of Navier-Stokes solutions on $\mathbb{R}^{3}$

(B) Existence and smoothness of Navier-Stokes solutions on $\mathbb{R}^{3} / \mathbb{Z}^{3}$

(C) Breakdown of Navier-Stokes solutions on $\mathbb{R}^{3}$

(C) Breakdown of Navier-Stokes solutions on $\mathbb{R}^{3} / \mathbb{Z}^{3}$

In this paper, we prove the statement (B) which can be alternatively formulated as follows:

Theorem 1.3. For every $u_{0} \in H_{\sigma}^{1}\left(\mathbb{T}^{3}\right)$ there exists a unique global in time strong solution $u \in L^{\infty}\left(0, \infty ; H_{\sigma}^{1}\left(\mathbb{T}^{3}\right)\right) \cap L^{2}\left(0, \infty ; H^{2}\left(\mathbb{T}^{3}\right)\right)$ of the Navier-Stokes equations. 
The method used to extend the solution into a global one is to prove that on an interval of strictly positive length $\left[t_{0}, t_{1}\right] \subset\left(0, T_{\max }\right)$ under a certain condition on $\sum_{k \in \mathbb{Z}^{3}}|\hat{u}(k, t)|$ among two possible ones:

$$
\underbrace{\sum_{|k|>m}|\hat{u}(k, t)| \leq \sum_{|k| \leq m}|\hat{u}(k, t)|}_{\text {condition } 1} \text { or } \underbrace{\sum_{|k| \leq m}|\hat{u}(k, t)| \leq \sum_{|k|>m}|\hat{u}(k, t)|}_{\text {condition } 2}
$$

the solution will be controlled in $H^{1}$ by a function defined in terms of time $t$, $\left\|\nabla u\left(t_{0}\right)\right\|_{L^{2}},\left\|u_{0}\right\|_{L^{2}}$ and a finite number $m$ (depending on the viscosity $\nu$ and $\left.\left\|\nabla u\left(t_{0}\right)\right\|_{L^{2}}\right)$, until reaching $t_{1}$. Otherwise, that is if condition 2 holds true $\forall t \in$ $\left[t_{0}, t_{1}\right]$, then the norm $\|\nabla u(t)\|_{L^{2}}^{2}$ is non-increasing on $\left[t_{0}, t_{1}\right]$. We continue then in this vein until reaching $T_{\max }$. To be more precise, we subdivide the interval $\left(0, T_{\max }\right)$ into a series of successive sub-intervals each of them is akin to $\left[t_{0}, t_{1}\right]$, i.e.: on each of them either condition 1 or 2 holds.

We quote the following two results, the proof of which is given in [9] and based on that of (Theorem $10.6[3]$ ).

Theorem 1.4. Let $u$ be a strong solution of the Navier-Stokes equations (NS) on the time interval $[0, T]$, with initial condition $u_{0} \in H^{1}$. Then for all $0<\varepsilon<T$ we have $u \in C\left([\varepsilon, T] ; H^{p}\right)$ for all $p \in \mathbb{N}$. In particular, for all $t \in[0, T)$ the function $u(t)$ is smooth with respect to the space variables.

The Theorem 1.4 together with the following lemma constitute a cornerstone in establishing the proof of Theorem 1.3.

Lemma 1.5. Let $u$ be a strong solution of the Navier-Stokes equations on the time interval $[0, T]$. Then for every $\varepsilon>0$, and all $p, l \in \mathbb{N}$ we have

$$
\partial_{t}^{l} u \in L^{\infty}\left(\varepsilon ; T ; H^{p}\right)
$$

The rest of the paper is dedicated to give the proof of Theorem 1.3.

\section{THE PROOF}

The analysis can be started by sketching the procedure through which the existence of local in time strong solution to $(N S)$ follows. To this end, let $P_{n}$ be the projection onto the Fourier modes of order up to $n \in \mathbb{N}$, that is

$$
P_{n}\left(\sum_{k \in \mathbb{Z}^{3}} \hat{\vartheta}_{k} e^{i x k}\right)=\sum_{|k| \leq n} \hat{\vartheta}_{k} e^{i x k}
$$

Let $u_{n}=P_{n} u$ be the solution to

$$
\left\{\begin{array}{l}
\partial_{t} u_{n}-\nu \Delta u_{n}+P_{n}\left[\left(u_{n} \cdot \nabla\right) u_{n}\right]=0, \quad(x, t) \in \mathbb{T}^{3} \times \mathbb{R}_{+} \\
\nabla \cdot u_{n}=0, \quad(x, t) \in \mathbb{T}^{3} \times \mathbb{R}_{+} \\
\left.u_{n}\right|_{t=0}(x)=P_{n}\left(u^{i n}\right)(x), \quad x \in \mathbb{T}^{3}
\end{array}\right.
$$


For some $T_{n}$, there exists a solution $u_{n} \in C^{\infty}\left(\left[0, T_{n}\right) \times \mathbb{T}^{3}\right)$ to this finite-dimensional locally-Lipschitz system of ODEs. We take the $L^{2}$-inner product of the first equation in $\left(N S_{n}\right)$ against $-\Delta u_{n}$ to obtain

$$
\begin{aligned}
\frac{1}{2} \frac{d}{d t}\left\|\nabla u_{n}(t)\right\|_{L^{2}}^{2} & +\nu\left\|\Delta u_{n}(t)\right\|_{L^{2}}^{2} \\
& \leq\left|\left\langle\left(u_{n} \cdot \nabla u_{n}\right), \Delta u_{n}\right\rangle_{L^{2}\left(\mathbb{T}^{3}\right)}\right| \\
& \leq\left\|u_{n}(t)\right\|_{L^{\infty}\left(\mathbb{T}^{3}\right)}\left\|\nabla u_{n}(t)\right\|_{L^{2}\left(\mathbb{T}^{3}\right)}\left\|\Delta u_{n}(t)\right\|_{L^{2}\left(\mathbb{T}^{3}\right)} \\
& \leq c\left\|u_{n}(t)\right\|_{H^{1}\left(\mathbb{T}^{3}\right)}^{1 / 2}\left\|u_{n}(t)\right\|_{H^{2}\left(\mathbb{T}^{3}\right)}^{1 / 2}\left\|\nabla u_{n}(t)\right\|_{L^{2}\left(\mathbb{T}^{3}\right)}\left\|\Delta u_{n}(t)\right\|_{L^{2}\left(\mathbb{T}^{3}\right)} \\
& \leq c\left\|\nabla u_{n}(t)\right\|_{L^{2}\left(\mathbb{T}^{3}\right)}^{3 / 2}\left\|\Delta u_{n}(t)\right\|_{L^{2}\left(\mathbb{T}^{3}\right)}^{3 / 2},
\end{aligned}
$$

where we used Hölder's inequality together with Agmon's inequality [1] and the Poincaré inequality.

Using Young's inequality with exponents 4 and $4 / 3$ yields

$$
\left|\left\langle\left(u_{n} \cdot \nabla u_{n}\right), \Delta u_{n}\right\rangle_{L^{2}\left(\mathbb{T}^{3}\right)}\right| \leq c\left\|\nabla u_{n}\right\|^{6}+\frac{\nu}{2}\left\|\Delta u_{n}(t)\right\|_{L^{2}}^{2},
$$

where $c$ is a positive constant that does not depend on $n$. It turns out that

$$
\frac{d}{d t}\left\|\nabla u_{n}(t)\right\|_{L^{2}}^{2}+\nu\left\|\Delta u_{n}(t)\right\|_{L^{2}}^{2} \leq c\left\|\nabla u_{n}\right\|^{6} .
$$

By comparing the function $\left\|\nabla u_{n}(t)\right\|_{L^{2}}^{2}$ with the solution of the ODE:

$$
\frac{d x}{d t}=c x^{3}, x(0)=\left\|\nabla u_{0}\right\|_{L^{2}}^{2},
$$

we infer that as long as $0 \leq t<\frac{1}{2 c\left\|\nabla u_{0}\right\|_{L^{2}}^{4}}$, the following holds

$$
\left\|\nabla u_{n}(t)\right\|_{L^{2}}^{2} \leq \underbrace{\frac{\left\|\nabla u_{0}\right\|_{L^{2}}^{2}}{\sqrt{1-2 c t\left\|\nabla u_{0}\right\|_{L^{2}}^{4}}}}_{\mathcal{W}(t)} .
$$

Estimate (2.1) tells us that an initial data as large as $\left\|\nabla u_{0}\right\|_{L^{2}}$ gives rise to a solution $u$ that would remain bounded on the interval $\left[0, T_{\max }\right)$ where $T_{\max } \sim \frac{1}{\left\|\nabla u_{0}\right\|_{L^{2}}^{4}}$. However, what happens after time $T_{\max }$ is unknown.

We turn now to the question of whether a local in time strong solution can be extended into a global solution. To this end, let us make estimates directly for $u$ instead of using the Galerkin approximation. We know that $u_{0}$ gives rise to a strong solution that exists at least on a certain time interval $\left[0, T_{\max }\right)$. On this time interval for each time $t \in\left(0, T_{\max }\right)$ we take the $L^{2}$-inner product of $(N S)$ against $-\Delta u$, we obtain

$$
\begin{aligned}
\frac{1}{2} \frac{d}{d t}\|\nabla u(t)\|_{L^{2}}^{2} & +\nu\|\Delta u(t)\|_{L^{2}}^{2} \\
& \leq\left|\langle(u \cdot \nabla u), \Delta u\rangle_{L^{2}\left(\mathbb{T}^{3}\right)}\right| \\
& \leq\|u(t)\|_{L^{\infty}\left(\mathbb{T}^{3}\right)}\|\nabla u(t)\|_{L^{2}\left(\mathbb{T}^{3}\right)}\|\Delta u(t)\|_{L^{2}\left(\mathbb{T}^{3}\right)} .
\end{aligned}
$$

The Fourier expansion of $u(x, t)$ is given by

$$
u(x, t)=\sum_{k \in \mathbb{Z}^{3}} \hat{u}(k, t) e^{i k x} .
$$


For a certain number $m$ (to be discussed later on), we have

$$
\begin{aligned}
\|u(t)\|_{L^{\infty}\left(\mathbb{T}^{3}\right)} & \leq \sum_{k \in \mathbb{Z}^{3}}|\hat{u}(k, t)| \\
& =\sum_{|k| \leq m}|\hat{u}(k, t)|+\sum_{|k|>m}|\hat{u}(k, t)| .
\end{aligned}
$$

Two possible natural cases may occur. The first is when the major amount of energy at the instant $t$ is quantized in low-frequency components. This case can be represented by

$$
\sum_{|k|>m}|\hat{u}(k, t)| \leq \sum_{|k| \leq m}|\hat{u}(k, t)|
$$

The second case is when the major amount of energy at time $t$ is quantized in high-frequency components. That is to say

$$
\sum_{|k| \leq m}|\hat{u}(k, t)| \leq \sum_{|k|>m}|\hat{u}(k, t)|
$$

We state here the Agmon's inequality [1] which reads:

$$
\sum_{k \in \mathbb{Z}^{3}}|\hat{u}(k, t)| \leq c\|u(t)\|_{H^{1}\left(\mathbb{T}^{3}\right)}^{1 / 2}\|u(t)\|_{H^{2}\left(\mathbb{T}^{3}\right)}^{1 / 2}
$$

By Theorem 1.4 we have $u \in C\left(\left[0, T_{\max }\right) ; H^{1}\right)$ and $C\left(\left(0, T_{\max }\right) ; H^{2}\right)$, then by a continuity argument one can always find at least a small interval of strictly positive length $\left[t_{0}, t_{1}\right] \subset\left(0, T_{\max }\right)$ on which either $(2.2)$ or $(2.3)$ occurs for any positive number $m$. Since $\left[t_{0}, t_{1}\right]$ will serve as a test interval to examine case (2.2) and case (2.3), one can choose without loss of generality $t_{0}$ very close to the instant zero. To be more precise, let $t_{0}$ be the instant of time immediately after $t=0$, there exists $t_{1}>t_{0}$ such that we have either (2.2) for all $t \in\left[t_{0}, t_{1}\right]$ or $(2.3)$ for all $t \in\left[t_{0}, t_{1}\right]$. We need also to make use of Lemma 1.5, which combined with the fact that $\partial_{t}|\hat{u}(k, t)| \leq\left|\partial_{t} \hat{u}(k, t)\right|$ yields

$$
\partial_{t} \sum_{k \in \mathbb{Z}^{3}}|\hat{u}(k, t)| \in L^{\infty}\left(\varepsilon, T_{\text {max }} ; H^{p}\right), \forall p \in \mathbb{N}
$$

Property (2.4) is useful because it assures the smoothness of $\sum_{k \in \mathbb{Z}^{3}}|\hat{u}(k, t)|$ with respect to time and hence that of $\sum_{|k| \leq m}|\hat{u}(k, t)|$ and $\sum_{|k|>m}|\hat{u}(k, t)|$. This prevents the abrupt bends of the function $t \mapsto F_{m}(t)=\sum_{|k| \leq m}|\hat{u}(k, t)|-\sum_{|k|>m}|\hat{u}(k, t)|$. Let us now discuss both cases on $\left[t_{0}, t_{1}\right]$. To be more precise, if (2.2) holds true on $\left[t_{0}, t_{1}\right]$ what will happen and if $(2.3)$ holds true on $\left[t_{0}, t_{1}\right]$ what will happen.

Case 1: 
By using (2.2), the Cauchy-Schwarz inequality and Young's inequality, we get

$$
\begin{aligned}
\frac{1}{2} \frac{d}{d t}\|\nabla u(t)\|_{L^{2}}^{2} & +\nu\|\Delta u(t)\|_{L^{2}}^{2} \\
& \leq 2 \sum_{|k| \leq m}|\hat{u}(k, t)|\|\nabla u(t)\|_{L^{2}\left(\mathbb{T}^{3}\right)}\|\Delta u(t)\|_{L^{2}\left(\mathbb{T}^{3}\right)} \\
& \leq 2\left(\sum_{|k| \leq m} 1\right)^{1 / 2}\left(\sum_{|k| \leq m}|\hat{u}(k, t)|^{2}\right)^{1 / 2} \\
& \times\|\nabla u(t)\|_{L^{2}\left(\mathbb{T}^{3}\right)}\|\Delta u(t)\|_{L^{2}\left(\mathbb{T}^{3}\right)} \\
& \leq 2\left(\sum_{|k| \leq m} 1\right)^{1 / 2}\|u(t)\|_{L^{2}}\|\nabla u(t)\|_{L^{2}\left(\mathbb{T}^{3}\right)}\|\Delta u(t)\|_{L^{2}\left(\mathbb{T}^{3}\right)} \\
& \leq C(m)\|u(t)\|_{L^{2}}^{2}\|\nabla u(t)\|_{L^{2}\left(\mathbb{T}^{3}\right)}^{2}+\frac{\nu}{2}\|\Delta u(t)\|_{L^{2}\left(\mathbb{T}^{3}\right)}^{2},
\end{aligned}
$$

where $C(m)=\frac{2 \sum_{|k| \leq m} 1}{\nu}$. By using the energy inequality for weak solutions (1.1) and dropping the viscous term from both sides above, we obtain

$$
\frac{d}{d t}\|\nabla u(t)\|_{L^{2}}^{2} \leq 2 C(m)\left\|u_{0}\right\|_{L^{2}}^{2}\|\nabla u(t)\|_{L^{2}\left(\mathbb{T}^{3}\right)}^{2} .
$$

The Gronwall's inequality yields

$$
\|\nabla u(t)\|_{L^{2}}^{2} \leq\left\|\nabla u\left(t_{0}\right)\right\|_{L^{2}}^{2} \exp \left\{2 t C(m)\left\|u_{0}\right\|_{L^{2}}^{2}\right\} \text {, for all } t \in\left[t_{0}, t_{1}\right] .
$$

Case 2:

By using (2.3) and the Cauchy-Schwarz inequality we infer that

$$
\begin{aligned}
\frac{1}{2} \frac{d}{d t}\|\nabla u(t)\|_{L^{2}}^{2} & +\nu\|\Delta u(t)\|_{L^{2}}^{2} \\
& \leq 2 \sum_{|k|>m}|\hat{u}(k, t)|\|\nabla u(t)\|_{L^{2}\left(\mathbb{T}^{3}\right)}\|\Delta u(t)\|_{L^{2}\left(\mathbb{T}^{3}\right)} \\
& =2 \sum_{|k|>m}|k|^{-2}|k|^{2}|\hat{u}(k, t)|\|\nabla u(t)\|_{L^{2}\left(\mathbb{T}^{3}\right)}\|\Delta u(t)\|_{L^{2}\left(\mathbb{T}^{3}\right)} \\
& \leq 2\left(\sum_{|k|>m}|k|^{-4}\right)^{1 / 2}\left(\sum_{|k|>m}|k|^{4}|\hat{u}(k, t)|^{2}\right)^{1 / 2} \\
& \times\|\nabla u(t)\|_{L^{2}\left(\mathbb{T}^{3}\right)}\|\Delta u(t)\|_{L^{2}\left(\mathbb{T}^{3}\right)} .
\end{aligned}
$$

Since $\sum_{|k|>m}|k|^{4}|\hat{u}(k, t)|^{2} \leq \sum_{k \in \mathbb{Z}^{3}}|k|^{4}|\hat{u}(k, t)|^{2}=\|\Delta u(t)\|_{L^{2}\left(\mathbb{T}^{3}\right)}^{2}$, it turns out

$$
\frac{1}{2} \frac{d}{d t}\|\nabla u(t)\|_{L^{2}}^{2}+\left\{\nu-2 c(m)\|\nabla u(t)\|_{L^{2}}\right\}\|\Delta u(t)\|_{L^{2}}^{2} \leq 0,
$$

where $c(m)=\left(\sum_{|k|>m}|k|^{-4}\right)^{1 / 2}$. Notice that $\lim _{m \rightarrow \infty} c(m)=0$, then one can choose the number $m$ such that

$$
c(m)<\frac{\nu}{2}\left\|\nabla u\left(t_{0}\right)\right\|_{L^{2}\left(\mathbb{T}^{3}\right)}^{-1} .
$$


GLOBAL EXISTENCE OF STRONG SOLUTION TO 3D PERIODIC NAVIER-STOKES EQUATION\$

In such a way, the factor $\left\{\nu-2 c(m)\|\nabla u(t)\|_{L^{2}\left(\mathbb{T}^{3}\right)}\right\}$ would still positive at least over a short interval of time $\left[t_{0}, \tau_{1}\right] \subset\left[t_{0}, t_{1}\right]$. Consequently, it turns out that

$$
\frac{d}{d t}\|\nabla u(t)\|_{L^{2}\left(\mathbb{T}^{3}\right)}^{2} \leq 0, \text { and }\|\nabla u(t)\|_{L^{2}\left(\mathbb{T}^{3}\right)} \leq\left\|\nabla u\left(t_{0}\right)\right\|_{L^{2}\left(\mathbb{T}^{3}\right)} \text { for all } t \in\left[t_{0}, \tau_{1}\right]
$$

But as $\|\nabla u(t)\|_{L^{2}\left(\mathbb{T}^{3}\right)}$ is continuous on $\left[t_{0}, t_{1}\right]$, we obtain

$$
\|\nabla u(t)\|_{L^{2}\left(\mathbb{T}^{3}\right)} \leq\left\|\nabla u\left(t_{0}\right)\right\|_{L^{2}\left(\mathbb{T}^{3}\right)} \text { for all } t \in\left[t_{0}, t_{1}\right] .
$$

Thus, the condition on $m$ has been determined successfully. To summarize, we have proved that there exists $t_{1}>t_{0}$ such that we have either

$$
\|\nabla u(t)\|_{L^{2}}^{2} \leq\left\|\nabla u\left(t_{0}\right)\right\|_{L^{2}}^{2} \exp \left\{2 t C(m)\left\|u_{0}\right\|_{L^{2}}^{2}\right\} \text {, for all } t \in\left[t_{0}, t_{1}\right]
$$

or

$$
\|\nabla u(t)\|_{L^{2}}^{2} \leq\left\|\nabla u\left(t_{0}\right)\right\|_{L^{2}}^{2}, \text { for all } t \in\left[t_{0}, t_{1}\right]
$$

where

$$
C(m)=\frac{2 \sum_{|k| \leq m} 1}{\nu}, \text { and } m=\inf \left\{p ;\left(\sum_{|k|>p}|k|^{-4}\right)^{1 / 2}<\frac{\nu}{4}\left\|\nabla u\left(t_{0}\right)\right\|_{L^{2}}^{-1}\right\} .
$$

Continuing in this vein, in the next interval we know already that $m$ must be as large as $c(m)<\frac{\nu}{2}\left\|\nabla u\left(t_{1}\right)\right\|_{L^{2}}^{-1}$ which guarantees by continuity that in case (2.3) the function $\|\nabla u(t)\|_{L^{2}}$ is non-increasing on this interval. There exists then $t_{2}>t_{1}$ such that

$$
\|\nabla u(t)\|_{L^{2}}^{2} \leq\left\|\nabla u\left(t_{1}\right)\right\|_{L^{2}}^{2} \exp \left\{2 t C(m)\left\|u_{0}\right\|_{L^{2}}^{2}\right\}, \text { for all } t \in\left[t_{1}, t_{2}\right]
$$

or

$$
\|\nabla u(t)\|_{L^{2}}^{2} \leq\left\|\nabla u\left(t_{1}\right)\right\|_{L^{2}}^{2} \text {, for all } t \in\left[t_{1}, t_{2}\right]
$$

where

$$
C(m)=\frac{2 \sum_{|k| \leq m} 1}{\nu}, \text { and } m=\inf \left\{p ;\left(\sum_{|k|>p}|k|^{-4}\right)^{1 / 2}<\frac{\nu}{4}\left\|\nabla u\left(t_{1}\right)\right\|_{L^{2}}^{-1}\right\} .
$$

Repeating this process as many times as needed to obtain $\left[t_{0}, T_{\max }-\varepsilon\right]=\cup_{j=0}^{N}\left[t_{j}, t_{j+1}\right]$ (where $\varepsilon>0$ is an arbitrary small constant and $\left[t_{j}, t_{j+1}\right]$ are successive intervals), such that for all $t \in\left[t_{j}, t_{j+1}\right]$ we have either

$$
\|\nabla u(t)\|_{L^{2}}^{2} \leq\left\|\nabla u\left(t_{j}\right)\right\|_{L^{2}}^{2} \exp \left\{2 t C(m)\left\|u_{0}\right\|_{L^{2}}^{2}\right\}
$$

or

$$
\|\nabla u(t)\|_{L^{2}}^{2} \leq\left\|\nabla u\left(t_{j}\right)\right\|_{L^{2}}^{2}
$$

where

$$
C(m)=\frac{2 \sum_{|k| \leq m} 1}{\nu}, \text { and } m=\inf \left\{p ;\left(\sum_{|k|>p}|k|^{-4}\right)^{1 / 2}<\frac{\nu}{4}\left\|\nabla u\left(t_{j}\right)\right\|_{L^{2}}^{-1}\right\} .
$$

We remark that the number $m$ may change from an interval $\left[t_{j}, t_{j+1}\right]$ to the next one $\left[t_{j+1}, t_{j+2}\right]$, but it remains finite as long as $\left\|\nabla u\left(t_{j+1}\right)\right\|_{L^{2}}$ is finite which is guaranteed by the estimates (2.6) or (2.7). This process would certainly control the norm $\|\nabla u(t)\|_{L^{2}}^{2}$ and rules out the blowup of $u$ in $H^{1}\left(\mathbb{T}^{3}\right)$ as $t$ approaches $T_{\max }$. Therefore, the solution $u$ can be extended into a global in time strong solution. 


\section{Discussion}

At this point, one may ask the question under which condition the norm $\|\nabla u(t)\|_{L^{2}}$ keeps decreasing for all positive time $t \in \mathbb{R}_{+}$. In fact, this is possible when the distribution of energy in the initial data is extremely unbalanced (i.e. $\sum_{|k| \leq m}|\hat{u}(k, 0)|<<$ $\left.\sum_{|k|>m}|\hat{u}(k, 0)|\right)$. In that case, by smoothness of the function $\sum_{k \in \mathbb{Z}^{*}}|\hat{u}(k, t)|$ with respect to time, condition (2.3) keeps for a long interval of time until potentially $\|\nabla u(t)\|_{L^{2}}$ satisfies the smallness condition of [5].

Another aspect to discuss here is the motivation behind choosing the instant $t_{0}$ very close to zero. In fact, by doing so one can ensure via (2.1) the closeness of $\left\|\nabla u\left(t_{0}\right)\right\|_{L^{2}}$ to $\left\|\nabla u_{0}\right\|_{L^{2}}$ while holding the necessary regularity $\left(u\left(t_{0}\right) \in H^{p}\left(\mathbb{T}^{3}\right)\right.$ for all $p \in \mathbb{N})$. This also guarantees the minimum worsening to $\left\|\nabla u\left(t_{1}\right)\right\|_{L^{2}}$ in case (2.2). However, it is needless to say that this was optional and that one can choose any instant $t_{0} \in\left[0, T_{\max }\right)$ as initial time.

\section{Conclusion}

We have already proved that the local in time strong solution to $(N S)$ can be extended to be global in time strong solution. This was done via making estimates to $u$ in $H^{1}$ on a series of time intervals requiring that the function $t \mapsto F_{m}(t)=\sum_{|k| \leq m}|\hat{u}(k, t)|-\sum_{|k|>m}|\hat{u}(k, t)|$ keeps its sign constant (either positive or negative) on each of them. It is worth noting that when the major amount of energy is located in high-frequency components (i.e. $F_{m}(t) \leq 0$ ), the norm $\|\nabla u(t)\|_{L^{2}}$ decreases with time. This is in fact consistent with the phenomenology of the turbulent cascade which states that energy is dissipated at the small scales (i.e. higher frequencies).

\section{REFERENCES}

[1] S. Agmon, Lectures on Elliptic Boundary Value Problems, Van Nostrand, New York, 1965.

[2] J. Y. Chemin, Remarques sur l'existence globale pour le système de Navier-Stokes incompressible. SIAM Journal on Mathematical Analysis 1992; 23:20-28

[3] P. Constantin and C. Foias, Navier-Stokes equations. University of Chicago Press, Chicago, IL (1988)

[4] C. Fefferman, Existence and smoothness of the Navier-Stokes equation. http://www.claymath.org, (2000).

[5] H. Fujita and T. Kato, On the Navier-Stokes initial value problem I. Archive for Rational Mechanics and Analysis 1964; 16: 269-315.

[6] E. Hopf, Über die Aufgangswertaufgave für die hydrodynamischen Grundliechungen. Math. Nachr. 4, 213-231 (1951)

[7] A. Kiselev, and O. A. Ladyzhenskaya, On the existence and uniqueness of the solution of the nonstationary problem for a viscous, incompressible fluid. Izv. Akad. Nauk SSSR. Ser. Mat. 21, 655-680 (1957)

[8] J. Leray, Essai sur le mouvement d'un liquide visqueux emplissant l'espace. Acta Math. 63, 193-248 (1934)

[9] J. Robinson, J. Rodrigo and W. Sadowski, The Three-Dimensional Navier-Stokes Equations: Classical Theory (Cambridge Studies in Advanced Mathematics). Cambridge: Cambridge University Press (2016). doi:10.1017/CBO9781139095143

Department of mathematics, Faculty of Mathematical, Physical and Natural, Sciences of Tunis, University of Tunis El Manar, Tunis 2092, Tunisia.

Email address: abdelkerim.chaabani@fst.utm.tn 